\title{
Dielectric Breakdown of Electromagnetic Metamaterials in the Mean-Field Approximation
}

\author{
Jeffrey Boksiner and Timothy Bocskor \\ Space and Terrestrial Communications Directorate \\ US Army RDECOM CERDEC, Fort Monmouth, NJ, 07703, USA
}

\begin{abstract}
Electromagnetic Metamaterials (MTMs) are artificial materials with novel electromagnetic properties not available in nature. MTMs typically consist of a homogeneous host material containing appropriately configured embedded compact inclusions. MTMs have the potential to enable significant improvement on performance of low-profile (i.e. microstrip) and conformal antennas, including reduction of antenna size and antenna coupling. One key limitation for MTM implementation is the possibility of dielectric breakdown from electrical stresses such as high ambient electric fields arising from the transmitter itself, lightning and atmospheric charges, precipitation static (p-static), and electrostatic discharge (ESD).

In this paper we investigate dielectric breakdown in the meanfield approximation. Dielectric breakdown is deemed to occur if the electric potential across an insulator exceeds a certain critical value, causing the insulator to become conductive and leading to failure of the insulator. Embedded conductive structures, such as those comprising MTMs, cause electric field enhancement near the metallic inclusion lowering the electric strength.

We calculate the detailed electric field distribution within the MTM and compare the peak values to a critical breakdown field. Using the mean-field theory, we replace each inclusion by an equivalent dipole. The effect of remaining dipoles is taken into account by an effective field. The polarizability is determined by a self-consistent solution for the effective field. We determine the detailed field in the vicinity of the inclusion by summing the effective field and the local field due to the inclusion.

We find that the presence of inclusions reduces the electric strength of the MTM in comparison to the electric strength of the pure host material. For a dilute MTM, the reduction depends mainly on the geometry of the inclusion. The reduction depends weakly on the concentration of the inclusions and is independent of the permittivity of the host material. The reduction may be significant even for very dilute MTMs and needs to be taken into account in practical applications. This work is an initial stage in CERDEC's effort to evaluate limitations to MTM parameters given the presence of intrinsic or environmental electromagnetic fields.
\end{abstract}

\section{INTRODUCTION}

Electromagnetic Metamaterials (MTMs) are artificial materials that have novel electromagnetic properties not available in nature [1]. MTM can have unique properties, such as enhanced permittivity, negative material constants, or reactive input impedance [2]-[7]. Typically, MTM consist of a homogeneous host material of moderate permittivity $\epsilon$ and permeability $\mu$, in which a matrix of appropriately configured compact inclusions is embedded. The inclusions could be arrayed in a periodic configuration or could be randomly distributed. Common configurations of periodic inclusions include spheres, rods, or split-ring resonators (SRRs).
MTMs may offer significant advantages in development of military antennas. These advantages include reduction of antenna size and suppression of surface waves. However, antennas need to be deployed in real platforms and must survive in harsh military environments. One key limitation for MTM implementation is the possibility of dielectric breakdown. Any dielectric insulating material is subject to variety of electrical stresses. These include:

- High ambient electric fields arising from the transmitter,

- Lightning and atmospheric charges,

- Precipitation static (p-static), and

- Electrostatic discharge (ESD).

We investigate the onset of dielectric breakdown as a function of the inclusion geometry and density for MTMs with the following inclusions: cylinders, spheres, ellipsoids, and disks. Dielectric breakdown occurs if the electric potential across an insulator exceeds a certain critical value, causing the insulator to become conductive and leading to failure of the insulator. There is a considerable body of research into breakdown of dielectric materials due to their industrial utility. In particular, physicists have studied the reduction in electric strength due to embedded metallic structures in the dielectric. Embedded conductive structures cause electric field enhancement near the metallic inclusion lowering the electric strength. The value of the breakdown field depends on various factors such as shape of the inclusions, their distribution, and their concentration.

The permittivity of an MTM depends on the average electric field in the MTM, while the reduction in dielectric breakdown strength depends on the peak electric field, so that the relationship between permittivity enhancement and the degradation of electric strength is not necessarily straightforward. The relationship is especially complex for MTMs designed to operate in resonant regions.

Reduction in electric strength may become an important limiting factor in MTM design for practical applications. In this paper we aim to begin to investigate this for dilute MTMs under quasi-static conditions. Our approach is to calculate the detailed electric field distribution within the MTM and compare the peak values of the field to the field that would exist in a homogeneous material. We assume that some form of dielectric breakdown occurs if the local electric field exceeds a critical value (the breakdown field). This is a useful model often adopted for investigation of statistics of dielectric breakdown [8]. Under this model, the ratio of the peak field 
in the MTM to the field in a homogeneous material represents the reduction of electric strength of the MTM in comparison to the homogeneous host material.

\section{OnE-Dimensional EXAMPLE}

We illustrate the impact of conductive inclusions with a simple one-dimensional example. Consider a simple parallel plate capacitor filled with a lossless dielectric $\epsilon$ and having plate separation $l$ as shown in Fig. 1a. Suppose there is a metallic inclusion of width $w$. Fig. $1 \mathrm{~b}$ shows the configuration. If a voltage $V$ is applied, in the absence of the inclusion the electric field is $E=V / l$. The capacitance is proportional to $\epsilon / l$. The effect of the inclusion is to shorten the spacing between the conductors so that the electric field becomes

$$
E^{\prime}=\frac{V}{l-w} .
$$

The capacitance of the circuit is increased as we now have two capacitors in series:

$$
C^{\prime} \propto \frac{1}{2} \frac{2 \epsilon}{l-w} \propto \frac{\epsilon}{l-w} .
$$

Thus, we can imagine the entire capacitor filled with an MTM having an effective dielectric constant given by:

$$
\epsilon_{\mathrm{eff}}=\epsilon \frac{l}{l-w} .
$$

The electric field inside the dielectric (the microscopic field) at a given applied voltage increases by a factor $\frac{l}{l-w}$, so that if $E_{b}$ denotes the critical field above which dielectric breakdown occurs, the breakdown voltage for the material with inclusions is reduced by the same factor $\frac{l}{l-w}$. This illustrates the basic feature that while conductive inclusions enhance the permittivity, they simultaneously degrade the electric strength of the MTM. In the one-dimensional case, the electric field is uniform, so the factors that provide the enhancement and the dielectric degradation are the same. In higher dimension, this may not be the case.

It is useful to look at the capacitor problem in a different way. We can replace the metallic inclusion by an equivalent dipole moment $p$ as shown in Fig. 1c, such that

$$
p=w Q^{\prime} .
$$

Note that the charge $Q^{\prime}$ on the capacitor with the inclusion increases over the charge $Q$ on the capacitor with homogeneous medium by the same factor $\frac{l}{l-w}$ as the electric field so that

$$
p=\frac{l w Q}{l-w} .
$$

This creates an equivalent polarization $P$ given by

$$
P=\frac{p}{l}=\frac{w Q}{l-w}=\frac{\epsilon}{4 \pi} \frac{w E}{l-w} .
$$

We can calculate the effective electric susceptibility $\chi_{e}$ due to the inclusion as

$$
\chi_{e}=\frac{P}{E}=\frac{\epsilon}{4 \pi} \frac{w}{l-w},
$$

and the corresponding effective permittivity is the sum of contributions of the molecular susceptibility of the original dielectric $\chi_{\mathrm{mol}}$ and the effective susceptibility of the inclusion, so that

$$
\epsilon_{\mathrm{eff}}=1+4 \pi \chi_{\mathrm{mol}}+4 \pi \chi_{e}=\epsilon+4 \pi \chi_{e}=\epsilon \frac{l}{l-w} .
$$

As would be expected, the field approach in Eq. 8 and the circuit approach in Eq. 3 yield the same result.

\section{A. General Features}

The one-dimensional capacitor example illustrates several general features that apply to more complex geometries. They are:

- While the electric field in the MTM is inhomogeneous, the average of the electric field over the sample size $\hat{E}$, which is the macroscopically measurable field, is equal to the applied field or external electric field $E_{\text {ext }}$.

- The electric field induces a polarization on the inclusions that depends on the geometry of the material and the permittivity of the host material.

- If the material has electric strength $E_{b}$, the reduction in electric strength (susceptibility to breakdown) is proportional to the ratio of the peak electric field $E_{p}$ to the average field $\hat{E}$. This quantity depends only on the MTM geometry and is independent of the permittivity of the host material. We define this quantity as

$$
\beta=\frac{E_{p}}{\hat{E}}
$$

For a one-dimensional capacitor we have

$$
\beta=\frac{l}{l-w}=\frac{1}{1-v}=\frac{1}{1-\bar{w}},
$$

where $v$ is the fraction of the volume being filled by the inclusion and $\bar{w}$ is the fraction of the linear distance across the capacitor occupied by the inclusion.

\section{MEAN FIELD THEORY}

We approach the problem of determining the effect of conductive inclusions using the same approach as used to derive the dielectric constant for materials. We assume that the MTM is placed in an external electric field $E_{\text {ext }}$ oriented along a particular direction. We calculate all quantities with respect to this direction. Generalization to the vector fields is straightforward. We assume that the inclusions are perfect conductors and the host dielectric is lossless. Also, we perform the calculation in free space since the quantity that defines the degradation in electric strength is independent of permittivity of the host material.

We replace each inclusion by an equivalent dipole. The effect of remaining dipoles is taken into account by an effective field. The polarizability is determined by a self-consistent solution for the effective field. This approach is well-known in solid-state physics leading. Its application to the determination of material constitutive relations is known as as effective medium theory. The effective medium theory is one of the 


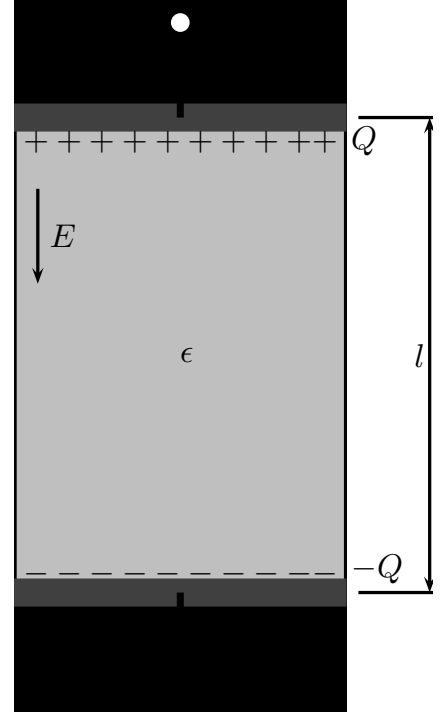

(a) Capacitor without inclusion

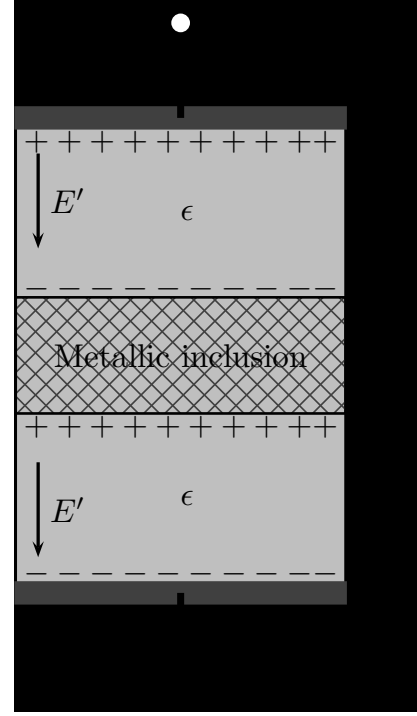

(b) With a metallic inclusion

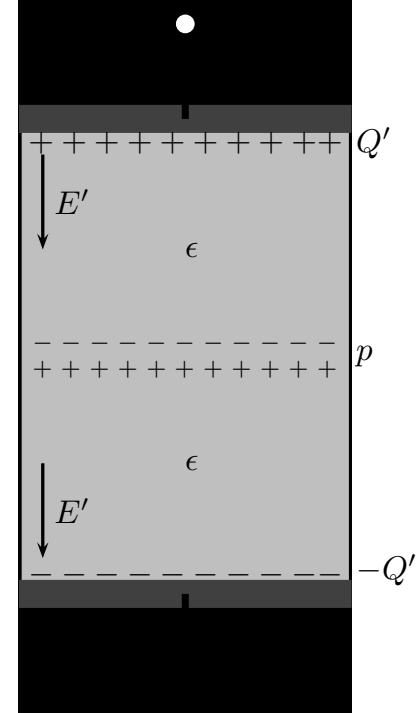

(c) With equivalent polarization.
Fig. 1. Illustration of a parallel-plate capacitor with a metallic inclusion where the metallic inclusion is replaced by the equivalent polarization

several approaches that have been developed to understand the average response of a heterogeneous medium. A thorough review is given by Markov [9]. Applications and extensions to MTMs is discussed by various authors such as [2], [6], [10]-[13]. The approach used here is valid under the following conditions:

- The inclusions are small compared to the wavelength,

- The inclusions are small compared to their separation (low concentration of inclusions),

- The distance between inclusions is small compared to the wavelength (quasistatic), and

- The inclusions are large compared to the molecular scale.

The key problem is the calculation of the local field that excites individual inclusions. A general formula that connects the local and average field valid for dilute inclusions is the
Lorenz-Lorentz formula [2], [14]

$$
E_{\mathrm{loc}}=\hat{E}+\frac{4 \pi}{3} P \text {. }
$$

The average polarization is

$$
P=N \alpha E_{\mathrm{loc}}
$$

where, $N$ is the concentration of inclusions, and $\alpha$ is the particle polarizability. Substituting into the Lorenz-Lorentz formula Eq. 11 we obtain the relation between the local field in the absence of the inclusion and the average field as

$$
E_{\mathrm{loc}}=\frac{1}{1-\frac{4 \pi}{3} N \alpha} \hat{E},
$$

and solving for the effective permittivity, we get the ClausiusMossotti relation [14], [15]

$$
\frac{\epsilon_{\text {eff }}-1}{\epsilon_{\text {eff }}+2}=\frac{4 \pi}{3} N \alpha .
$$

Equivalently, the mean field Clausius-Mossotti electric susceptibility is then [15]

$$
\chi_{e}=\frac{N \alpha}{1-\frac{4 \pi}{3} N \alpha} .
$$

The electric susceptibility or the dielectric constant is determined by the macroscopically measurable average field $\hat{E}$. However, the electric strength depends on the local peak field. Thus, we need to determine the peak field in the vicinity of each inclusion. This depends on the details of the shape of the inclusion. In general, the peak field must occur at the surface of the inclusion since the field decays away from the inclusion. The field normal to the inclusion is $E_{n}=4 \pi \sigma$, where $\sigma$ is the surface charge density. Thus, the peak field is given by

$$
E_{p}=\max E_{n}=4 \pi \max \sigma=4 \pi \sigma_{m}=\gamma E_{\mathrm{loc}}
$$

where $\gamma$ is a shape dependent coefficient between the uniform local field and the maximum surface charge density on the inclusion. We can combine Eq. 16 with Eq. 13 to obtain:

$$
E_{p}=\frac{\gamma}{1-\frac{4 \pi}{3} N \alpha} \hat{E}
$$

The susceptibility of the material to breakdown is determined in the dilute case by the shape of the inclusion and, to a lesser degree, by the density of the inclusions. However, we expect that the limiting case occurs for a spherical inclusion that has no mulipole components greater than a dipole.

\section{A. Three-Dimensional Spherical Inclusions}

Before proceeding to a general case we review a non-trivial three-dimensional example. We investigate a cubical array of spheres. The arrangement is shown in Fig. 2.

Sphere polarizability $\alpha$ in uniform field is given by [16]

$$
\alpha=R^{3} \text {. }
$$

The dipole moment of the sphere is $p=\alpha E_{\text {loc }}$. The surface charge density on a sphere in a uniform field $E$ is

$$
\sigma=\frac{3 E}{4 \pi} \cos \theta
$$




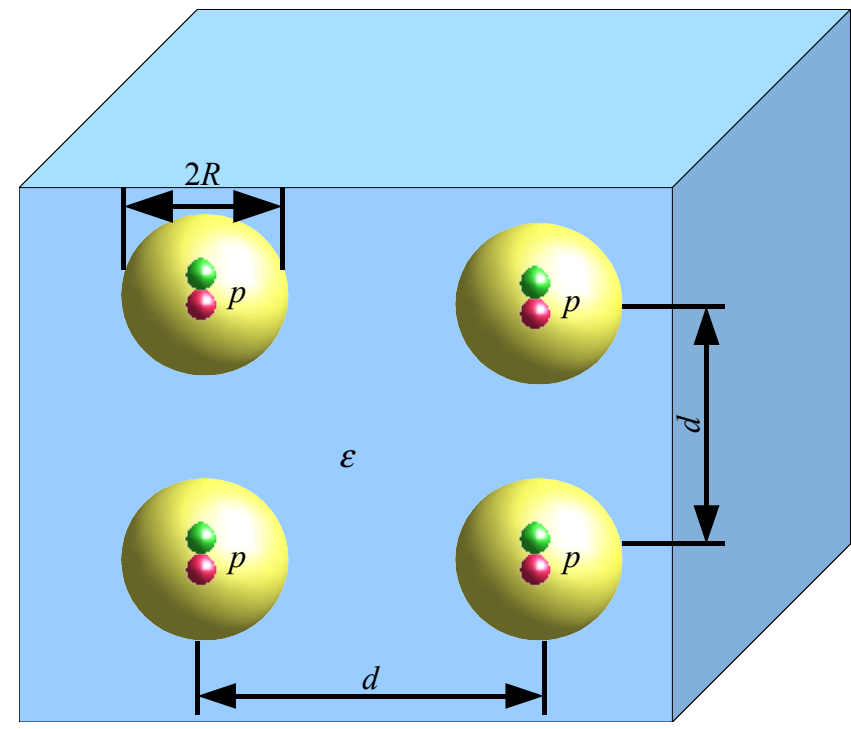

Fig. 2. Illustration of a cubical array of spheres and their equivalent polarization

where $\theta$ is the angle between $\vec{E}$ and $\vec{r}$. The maximum field at the surface of the sphere is

$$
E_{p}=4 \pi \sigma(\theta=0)=3 E_{\mathrm{loc}} .
$$

Therefore $\gamma=3$ and using Eq. 17 we can express the peak field as

$$
E_{p}=\frac{3}{1-\frac{4 \pi}{3} N \alpha} \hat{E}=\frac{3}{1-\frac{4 \pi R^{3}}{3 d^{3}}} \hat{E}=\frac{3}{1-v} \hat{E} \equiv \beta \hat{E},
$$

where $\beta$ represents the degradation in electric strength defined in Eq. 9 and $v$ is the fraction of the volume occupied by the inclusion. Note, that $v$ cannot reach unity even if the spheres were to touch each other, so as would be expected of the meanfield theory this result is not valid in case of dense packing of the spheres.

The result in Eq. 21 shows that for spherical inclusions the electric breakdown strength of an MTM is degraded by a factor of three or more in comparison to the pure host material even for a very dilute $(v \ll 1)$ material. Thus, if a layer of host material would have an electric strength of say $3 \mathrm{kV}$, the electric strength of the corresponding MTM would be reduced to $1 \mathrm{kV}$ or less. This reduction occurs even for very dilute materials since the presence of even a single inclusion will enhance the field in its vicinity.

\section{B. Two-Dimensional Cylindrical Inclusions}

In this section we review an important non-trivial twodimensional example. We investigate a square array of infinitely long cylinders. This "rodded" [17] media is an important MTM commonly used to create materials that exhibit negative permittivity.

Polarizability of a cylinder in uniform field is given by [16]

$$
\alpha=\frac{1}{2} R^{2} .
$$

The surface charge density on a cylinder in a uniform field $E$ is

$$
\sigma=\frac{E}{2 \pi} \cos \theta
$$

where $\theta$ is the angle between $\vec{E}$ and $\vec{r}$. The maximum field at the surface of the cylinder is

$$
E_{p}=4 \pi \sigma(\theta=0)=2 E_{\mathrm{loc}} .
$$

However, in this two-dimensional case, Eq. 15 must be modified for two-dimensional form of Clausius-Mossotti theory [10] as

$$
\chi_{e}^{2 \mathrm{D}}=\frac{N \alpha}{1-2 \pi N \alpha} .
$$

Therefore, with $\gamma=2$ and using Eq. 17 in two-dimensional form we can express the peak field for array of cylinders as

$$
E_{p}=\beta \hat{E}=\frac{2}{1-2 \pi N \alpha} \hat{E}=\frac{2}{1-\pi \frac{R^{2}}{d^{2}}} \hat{E}=\frac{2}{1-v} \hat{E} .
$$

The result in Eq. 26 shows that for cylindrical inclusions the electric breakdown strength of an MTM is degraded by a factor of two or more in comparison to the pure host material even for a very dilute material. Fig. 3 compares the behavior of the degradation for the examples in one, two, and three dimensions with cylindrical, or spherical inclusions as a function of $\bar{w}$, the ratio of the largest linear dimension of the inclusion in the direction of the applied field to the size of the unit cell. Note that the value of $\beta$ for the one-dimensional case represents the lowest limit of electric strength degradation for any configuration. Once, the value of $\beta$ for $2 \mathrm{D}$ and $3 \mathrm{D}$ cases falls below the values for 1D case, the mean-field theory is clearly invalid and the 1D result actually provides a better approximation.

\section{ELLIPTICAL INCLUSIONS}

We generalize the analysis of spherical inclusions to a general case of elliptical inclusions. Elliptical inclusions serve as models for a wide variety of practical MTM inclusions as shown in Table I. The analysis in this section is based on the discussion in Section 4 of Landau \& Lifshitz [16]. We consider a spheroid with the semiaxes $a, b$, and $c$. In a general case where the three semiaxes are different, the problem can be solved by using ellipsoidal coordinates. These are related to Cartesian coordinates as follows:

$$
\frac{x^{2}}{a^{2}+u}+\frac{y^{2}}{b^{2}+u}+\frac{z^{2}}{c^{2}+u}=1
$$

where $a>b>c$. The three roots of Eq. 27 are the ellipsoidal coordinates of point $x, y, z \mathrm{~s}$ follows:

$$
\begin{aligned}
\xi & \geq-c^{2}, \\
-c^{2} \geq \eta \geq-b^{2}, & \\
-b^{2} \geq \zeta & \geq-a^{2} .
\end{aligned}
$$




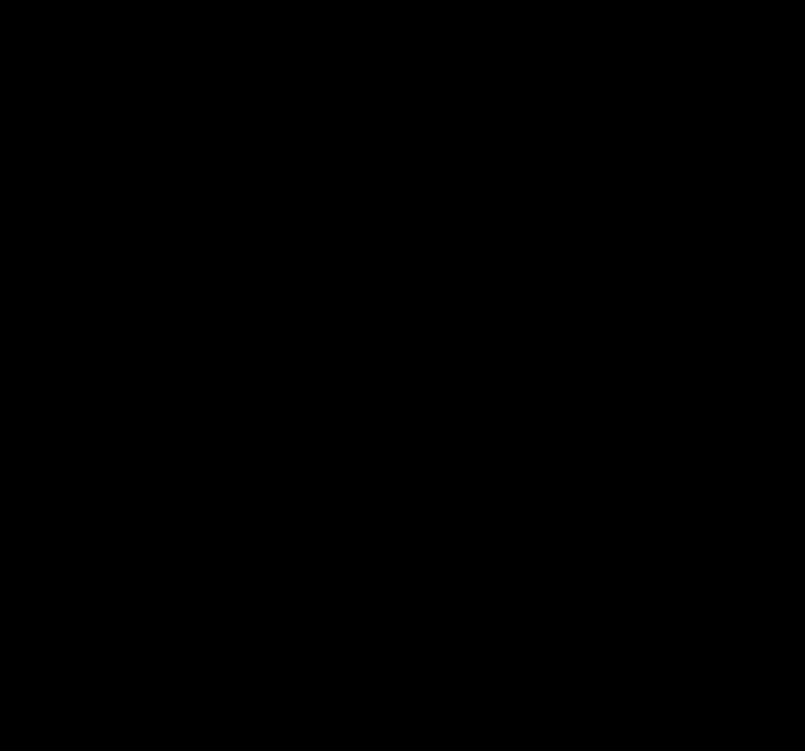

Fig. 3. Comparison of degradation in electric strength (susceptibility to breakdown) for symmetric inclusions of various dimensions as a function of the linear dimension $\bar{w}$ of the inclusion in the direction of the applied field normalized to the dimension of the unit cell.

The transformation from Cartesian coordinates is given by the following equations:

$$
\begin{aligned}
& x= \pm \sqrt{\frac{\left(\xi+a^{2}\right)\left(\eta+a^{2}\right)\left(\zeta+a^{2}\right)}{\left(b^{2}-a^{2}\right)\left(c^{2}-a^{2}\right)}} \\
& y= \pm \sqrt{\frac{\left(\xi+b^{2}\right)\left(\eta+b^{2}\right)\left(\zeta+b^{2}\right)}{\left(c^{2}-b^{2}\right)\left(a^{2}-b^{2}\right)}} \\
& z= \pm \sqrt{\frac{\left(\xi+c^{2}\right)\left(\eta+c^{2}\right)\left(\zeta+c^{2}\right)}{\left(a^{2}-c^{2}\right)\left(b^{2}-c^{2}\right)}}
\end{aligned}
$$

We do not consider the most general case of an ellipsoid with three different semiaxes, but focus on the case where the two semiaxes in the direction transverse to the applied field are equal. In this case there are two possibilities as follows:

1) Oblate spheroidal coordinates $(a=b>c)$ : The oblate spheroidal coordinates represent the case $b \rightarrow a$ and $\zeta \rightarrow$ $-a^{2}$. These coordinates are related to Cartesian coordinates as follows:

$$
\begin{aligned}
\frac{\rho^{2}}{a^{2}+u}+\frac{z^{2}}{c^{2}+u} & =1, \\
x^{2}+y^{2} & =\rho^{2} .
\end{aligned}
$$

The two roots of Eq. 30 lie in the range

$$
\begin{aligned}
& \xi \geq-c^{2}, \\
&-c^{2} \geq \eta \geq-a^{2} .
\end{aligned}
$$

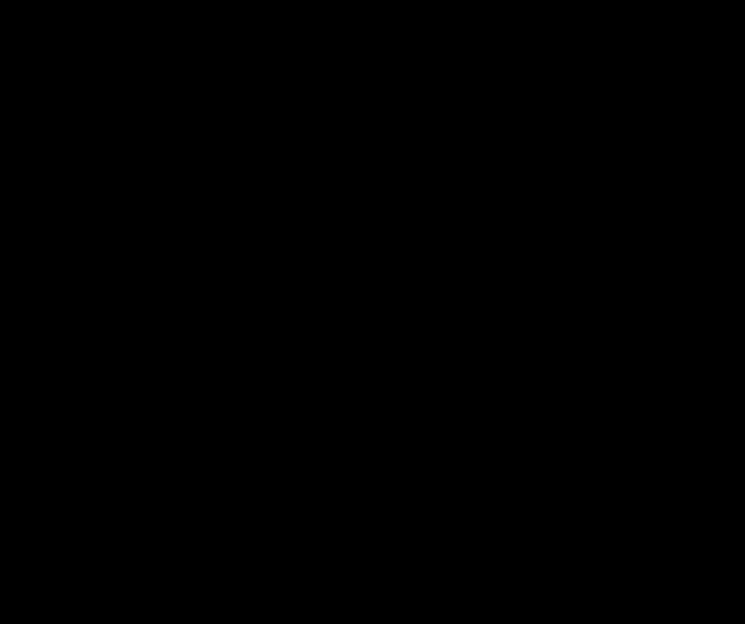

Fig. 4. Illustration of Oblate Spheroidal Coordinates

The transformation from cylindrical coordinates $z, \rho$ is given by the following equations:

$$
\begin{aligned}
& z= \pm \sqrt{\frac{\left(\xi+c^{2}\right)\left(\eta+c^{2}\right)}{c^{2}-a^{2}}}, \\
& \rho=\sqrt{\frac{\left(\xi+a^{2}\right)\left(\eta+a^{2}\right)}{a^{2}-c^{2}}} .
\end{aligned}
$$

Fig. 4 shows the oblate spheroidal coordinate system.

2) Prolate spheroidal coordinates $(a>b=c)$ : The prolate spheroidal coordinates represent the case $c \rightarrow b$ and $\eta \rightarrow-b^{2}$. The prolate spheroidal coordinates are related to Cartesian coordinates as follows:

$$
\begin{aligned}
\frac{x^{2}}{a^{2}+u}+\frac{\rho^{2}}{c^{2}+u} & =1, \\
z^{2}+y^{2} & =\rho^{2} .
\end{aligned}
$$

The two roots of Eq. 33 lie in the range

$$
\begin{aligned}
& \xi \geq-b^{2}, \\
&-b^{2} \geq \zeta \geq-a^{2} .
\end{aligned}
$$

The transformation from cylindrical coordinates $z, \rho$ is given by the following equations:

$$
\begin{aligned}
& x= \pm \sqrt{\frac{\left(\xi+a^{2}\right)\left(\zeta+a^{2}\right)}{a^{2}-b^{2}}}, \\
& \rho=\sqrt{\frac{\left(\xi+b^{2}\right)\left(\zeta+b^{2}\right)}{b^{2}-a^{2}}} .
\end{aligned}
$$

Fig. 5 shows this coordinate system.

\section{A. Ellipsoid in a Uniform Electric Field}

We consider an ellipsoid in a uniform field $E_{0}$ in the $x$ direction. The dipole moment of the ellipsoid due to the field in $\mathrm{x}$ direction is

$$
p_{x}=\alpha_{x} E_{0}=\frac{V}{4 \pi n^{(x)}} E_{0}
$$




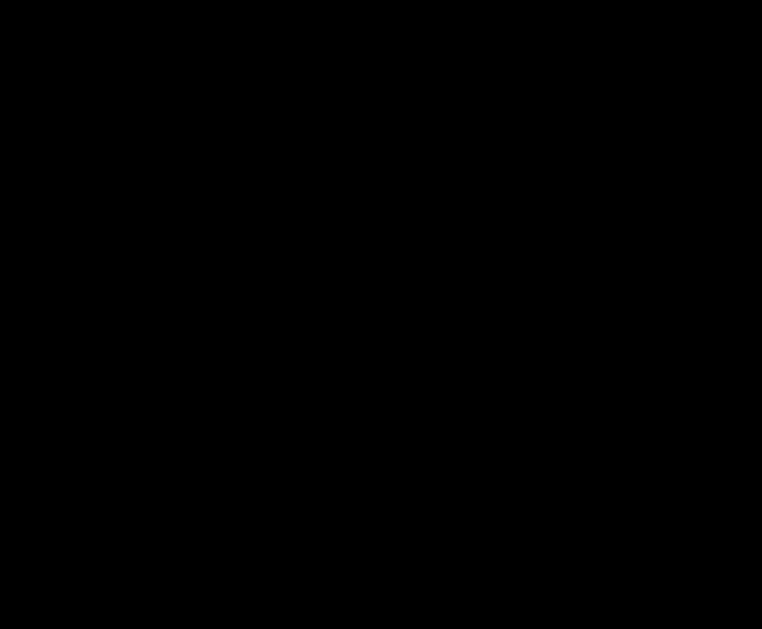

Fig. 5. Illustration of Prolate Spheroidal Coordinates

TABLE I

CORRESPONDENCE OF TYPICAL MTM INCLUSIONS TO ELLIPSOIDS

\begin{tabular}{|c|c|c|c|}
\hline \multicolumn{2}{|c|}{ Inclusion } & \multicolumn{2}{c|}{ Approximating Shape } \\
\hline Description & $\begin{array}{c}\text { Relevant } \\
\text { Dimension(s) }\end{array}$ & Shape & $\begin{array}{c}\text { Relevant } \\
\text { Ellipsoidal Parameters }\end{array}$ \\
\hline Sphere & Radius $(R)$ & Sphere & $a=b=c$ \\
Rods & Radius $(R)$ & Cylinder & $b=c, a \rightarrow \infty$ \\
SRR & Radius $(R)$ & Oblate & $a=b=R$, \\
& Thickness $(t)$ & Ellipsoid & $c \approx t$ \\
Stick & Length $(l)$ & Prolate & $a=l$, \\
& Radius $(R)$ & Ellipsoid & $b=c \approx R$ \\
\hline
\end{tabular}

where the volume $V$ is $V=\frac{4}{3} \pi a b c$, and $n^{(x)}$ is the depolarizing factor. The general definition for depolarizing factors $n^{(x)}, n^{(y)}$, and $n^{(z)}$ is

$$
\begin{aligned}
& n^{(x)}=\frac{1}{2} a b c \int_{0}^{\infty} \frac{d s}{\left(s+a^{2}\right) R_{s}}, \\
& n^{(y)}=\frac{1}{2} a b c \int_{0}^{\infty} \frac{d s}{\left(s+b^{2}\right) R_{s}}, \\
& n^{(z)}=\frac{1}{2} a b c \int_{0}^{\infty} \frac{d s}{\left(s+c^{2}\right) R_{s}} .
\end{aligned}
$$

The depolarizing factors obey the sum rule

$$
n^{(x)}+n^{(y)}+n^{(z)}=1
$$

The electric field on the surface is normal to the ellipsoid and is given by:

$$
E_{n}=\frac{\nu_{x}}{n^{(x)}} E_{0}
$$

where

$$
\nu_{x}=\left.\frac{x}{2 a^{2} h_{1}}\right|_{\xi=0}
$$

and

$$
h_{1}=\frac{\sqrt{(\xi-\eta)(\xi-\zeta)}}{2 \sqrt{\left(\xi+a^{2}\right)\left(\xi+b^{2}\right)\left(\xi+c^{2}\right)}} .
$$

Similar expressions apply for other Cartesian components. Therefore

$$
\gamma_{i}=\frac{\nu_{i}}{n^{(i)}},
$$

where $i$ denotes one of the Cartesian components $x, y, z$.

1) Prolate Spheroid: For a prolate spheroid $(a>b=c)$ of eccentricity

$$
e=\sqrt{1-\frac{b^{2}}{a^{2}}}
$$

the depolarizing factors are

$$
\begin{aligned}
n^{(x)} & =\frac{1-e^{2}}{2 e^{3}}\left(\ln \frac{1+e}{1-e}-2 e\right), \\
n^{(y)}=n^{(z)} & =\frac{1}{2}\left(1-n^{(x)}\right) .
\end{aligned}
$$

The factors $\nu$ are

$$
\begin{gathered}
\nu_{x}=\frac{a}{2 a^{2} \frac{1}{2 a}}=1, \\
\nu_{y}=\nu_{z}=\frac{b}{2 a^{2} \frac{1}{2 b}}=\frac{b^{2}}{a^{2}}=1-e^{2}
\end{gathered}
$$

2) Oblate Spheroid: For a oblate spheroid $(a=b>c)$ of eccentricity

$$
\begin{aligned}
e & =\sqrt{\frac{a^{2}}{c^{2}}-1} \\
n^{(z)} & =\frac{1+e^{2}}{2 e^{3}}\left(e-\tan ^{-1} e\right), \\
n^{(x)}=n^{(y)} & =\frac{1}{2}\left(1-n^{(z)}\right) .
\end{aligned}
$$

The factors $\nu$ are

$$
\begin{aligned}
\nu_{x}=\nu_{y} & =1, \\
\nu_{z} & =\frac{a}{2 a^{2} \frac{1}{2 c}}=\frac{c}{a}=\frac{1}{\sqrt{e^{2}+1}}
\end{aligned}
$$

3) Sphere: We can recheck our results for a sphere $a=$ $b=c$. The depolarizing factor is $\frac{1}{3}$, the $\nu$ s are unity. This recovers our previous result.

\section{B. Implication}

We see from the expression for the peak electric field Eq. 39 that the peak field is independent of the volume of the inclusion and depends only on the shape of the inclusion. Figs. 6 and 7 show the dependence of the factor $\gamma$ on the ellipsoid eccentricity. We see that the peak field for an infinitely thin needle or disk becomes infinite if the field is along the needle or the disk. This is typical for very sharp points. In reality, the magnitude of the field would be limited by the finite conductivity of the inclusion and the finite resistivity of the dielectric. 


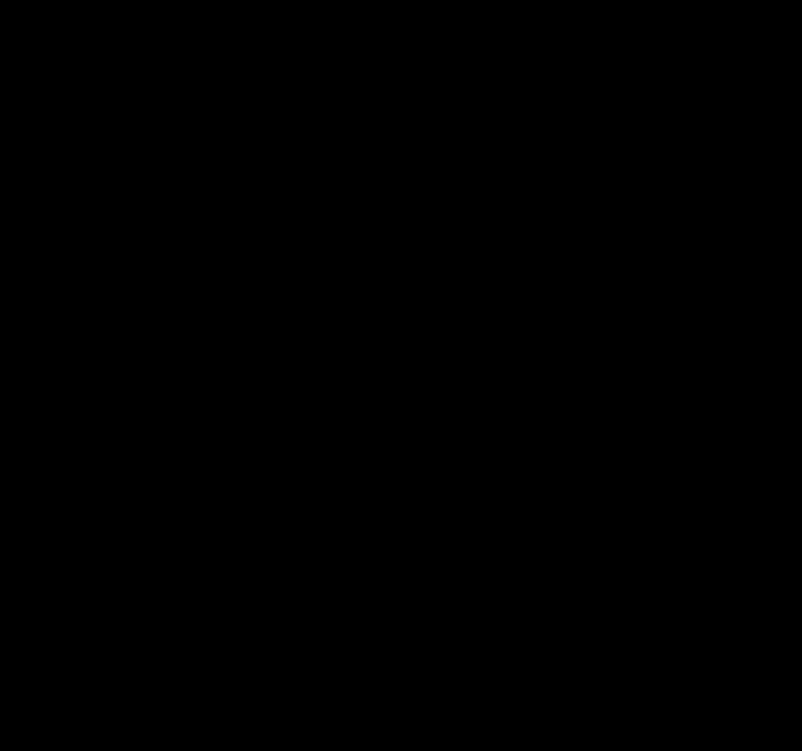

Fig. 6. The factors $\gamma$ as a function of eccentricity for prolate ellipsoid

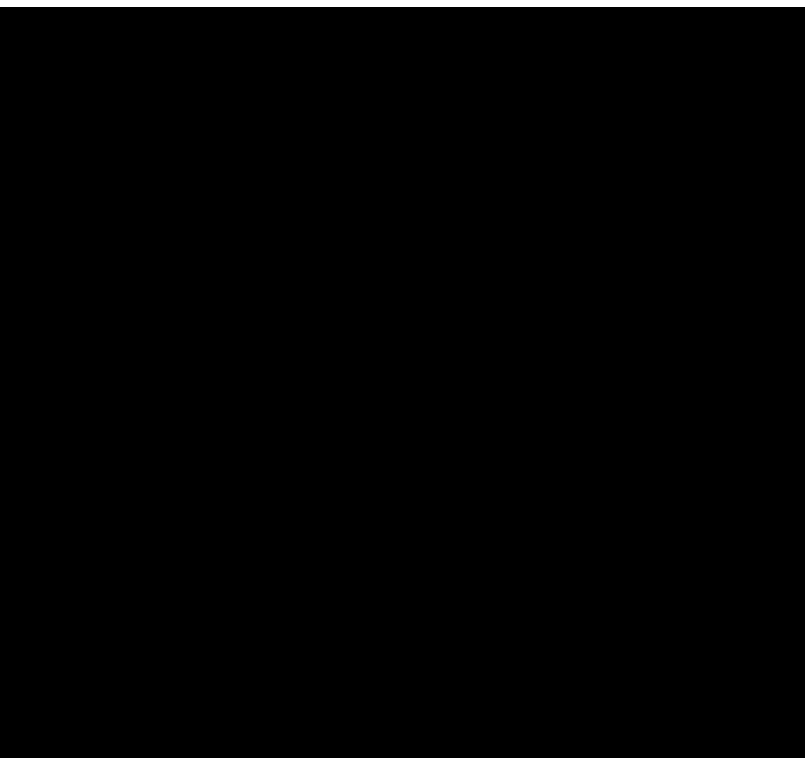

Fig. 7. The factors $\gamma$ as a function of eccentricity for oblate ellipsoid

\section{CONCLUSIONS}

An MTM consists of conductive inclusions in an otherwise insulating host material. The presence of conductive inclusions needed to create an MTM degrade the electric strength of the MTM in comparison to the pure host material. The degradation depends on the shape of the inclusion and the direction of the applied field. We have derived the expressions for the degradation by calculating the peak field at the surface of the inclusion for dilute MTMs. The field distribution will change in densely packed MTMs leading to different behavior of the electric strength. In general, we would expect the electric strength to degrade to low values as the density of inclusions increases.
Our analysis is based on assumption that dielectric breakdown occurs at a certain critical field. This is a useful assumption to help understand the effects of MTM inclusions, however, electric strength of real insulators also depends on other factors including the type of applied source, the waveform of the applied voltage, etc. In any case, it is important to remember that electric strength is an important engineering property of a material and must be considered in practical MTM applications.

\section{ACKNOWLEDGMENTS}

The authors thank Dr. Mahbub Hoque and Dr. Arthur Ballato for encouraging this work. The authors also thank the anonymous reviewers for helpful comments.

\section{REFERENCES}

[1] C. Caloz and T. Itoh, Electromagnetic Metamaterials: Transmission Line Theory and Microwave Applications. New Jersey: Wiley-Interscience, 2006.

[2] S. Tretyakov, Analytical Modeling in Applied Electromagnetics. Norwood MA: Artech House, 2003.

[3] G. V. Eleftheriades and K. G. Balmain, Negative-Refraction Metamaterials. New Jersey: IEEE Press, Wiley-Interscience, 2005.

[4] R. Marques, F. Martin, and M. Sorolla, Metamaterials with negative parameters: theory, design and microwave applications. Hoboken NJ: John Wiley \& Sons, 2008.

[5] J. D. Joannopoulos, R. D. Meade, and J. N. Winn, Photonic Crystals: Molding the Flow of Light. Princeton NJ: Princeton University Press, 1995.

[6] A. K. Sarychev and V. M. Shalaev, Electrodynamics of Metamaterials. Singapore: World Scientific, 2007.

[7] E. Shamonina and L. Solymar, "Metamaterials: How the subject started," Metamaterials, vol. 1, no. 1, pp. 12-18, Feb 2007.

[8] J. Boksiner and P. Leath, "Dielectric breakdown in media with defects," Phys. Rev. E, vol. 57, no. 3, p. 3531, Mar 1998.

[9] K. Z. Markov, Heterogeneous Media: Modelling and Simulation. Boston MA: Birkhauser, 1999.

[10] R. E. Collin, Field Theory of Guided Waves. New York NY: IEEE Press, 1991.

[11] D. R. Smith and J. B. Pendry, "Homogenization of metamaterials by field averaging," J. Opt. Soc. Am. B, vol. 23, no. 3, pp. 391-403, Mar 2006.

[12] J. M. Pitarke, F. J. García-Vidal, and J. B. Pendry, "Effective electronic response of a system of metallic cylinders," Phys. Rev. B, vol. 57, no. 24, pp. 15 261-15 266, Jun 1998

[13] W. M. Merrill, R. E. Diaz, M. M. LoRe, M. C. Squires, and N. G. Alexopoulos, "Effective medium theories for artificial materials composed of multiple sizes of spherical inclusions in a host continuum," IEEE Trans. Antennas Propagat., vol. 47, no. 1, pp. 142-148, Jan 1999.

[14] J. D. Jackson, Classical Electrodynamics. New York: John Wiley \& Sons, 1975.

[15] S. Sivasubramanian, A. Widom, and Y. Srivastava. (2002, Sep) Thermal superradiance and the Clausius-Mossotti Lorentz-Lorenz equations.

[16] L. Landau, E. Lifshitz, and L. Pitaevskii, Electrodynamics of Continuous Media. Tarrytown NY: Pergamon Press, 1984.

[17] W. Rotman, "Plasma simulation by artificial dielectrics and parallel-plate media," Antennas and Propagation, IRE Transactions on, vol. 10, no. 1, pp. 82-95, January 1962. 